\title{
The Life Predicting Calculations in Whole Process Realized by Calculable Materials Constants from short Crack to Long Crack Growth Process
}

\author{
Yangui Yu ${ }^{1,2}$ \\ ${ }^{1}$ Zhejiang Guangxin New Technology Application Academy of Electromechanical and Chemical Engineering, Hangzhou, China \\ ${ }^{2}$ Wenzhou University, Wenzhou, China
}

Email address:

gx_yyg@126.com; ygyu@vip.sina.com.cn

\section{To cite this article:}

Yangui Yu. The Life Predicting Calculations in Whole Process Realized by Calculable Materials Constants from short Crack to Long Crack Growth Process. International Journal of Materials Science and Applications. Vol. 4, No. 2, 2015, pp. 83-95.

doi: $10.11648 /$ j.ijmsa.20150402.13

\begin{abstract}
To use the theoretical approach, to adopt the simple stress-, or strain-parameter method, by means of the conventional material constants, to establish some new calculation models in whole crack growth process for elastic-plastic steels, which are the equations of the driving forces and the life predictions from micro to macro crack; To provide yet several crack growth-rate-linking-equations and life calculating expressions in whole process, for which are under different loading conditions in high cycle and low cycle fatigue. For key parameters $A_{1}, A_{2}$ and $B_{2}$ have proposed some new concept, and to define their physical and geometrical meanings. For the transition crack size from crack forming stage to crack growth stage, provide concrete calculation processes and methods. Thereby to realized the lifetime predicting calculations in whole process based on traditional calculable material parameters. The purpose is to try to make the modern fatigue-fracture discipline depended on tests become gradually calculable subjects as the traditional material mechanics. So that will be having practical significances for saving testing manpower and funds, for promoting applying and development relevant disciplines.
\end{abstract}

Keywords: Elastic-Plastic Materials, Fatigue Fracture, Crack Propagation Modeling, Low Cycle Fatigue, High Cycle Fatigue, Lifetime Prediction

\section{Introduction}

As everyone knows for the traditional material mechanics, that is a calculable subject, and has made valuable contributions for every industrial engineering designs and calculations. But it cannot accurately calculate the life problems for some structures when it is pre-existing flaws and under repeated loading. In that it has no to contain such calculable parameters as the crack variable $a$ or as the damage variable $D$ in their calculating models. On the other hand, inside the fracture mechanics and the damage mechanics, due to there are these variables, so they can just calculate above problems. But nowadays latter these disciplines are all subjects mainly depended on fatigue, damage and fracture tests.

Author thinks, in the mechanics and the engineering fields, in which are also to exist such scientific principles of similar to genetic and clone technology in life science. Author has done some of works used the theoretical approach as above the similar principles [1-8]. For example, for some strength calculation models from micro to macro are provided by reference [1], for some damage growth rate calculation models from micro to macro damage growth are proposed by references [2-8] which are models in each stage even in whole process, under different loading conditions. Two years ago, in order to do the lifetime calculations in whole process on fatigue-damage-fracture for an engineering structure, author was by means of Google Scholar to search the lifetime prediction models, as had been no found for this kind of calculation equations. After then, author continues to research this item, and bases on was provided and now is complemented on the comprehensive figure 1 of material behaviors [3]; still applies above genetic principles, to study and analyze data in references, thereby to provide some new calculable models for 
the new crack growth driving force and for the lifetime predictions. Try to make the fracture mechanics, step by step become calculable disciplines as the material mechanics. That way, may be having practical significances for decrease experiments, stint man powers and funds, for promoting engineering applying and developing to relevant disciplines.

\section{The Life Prediction Calculations for Elastic-Plastic Materials Containing Pre-Flaws}

\subsection{The Life Prediction Calculations in Short Crack Growth Process (Called the First Stage)}

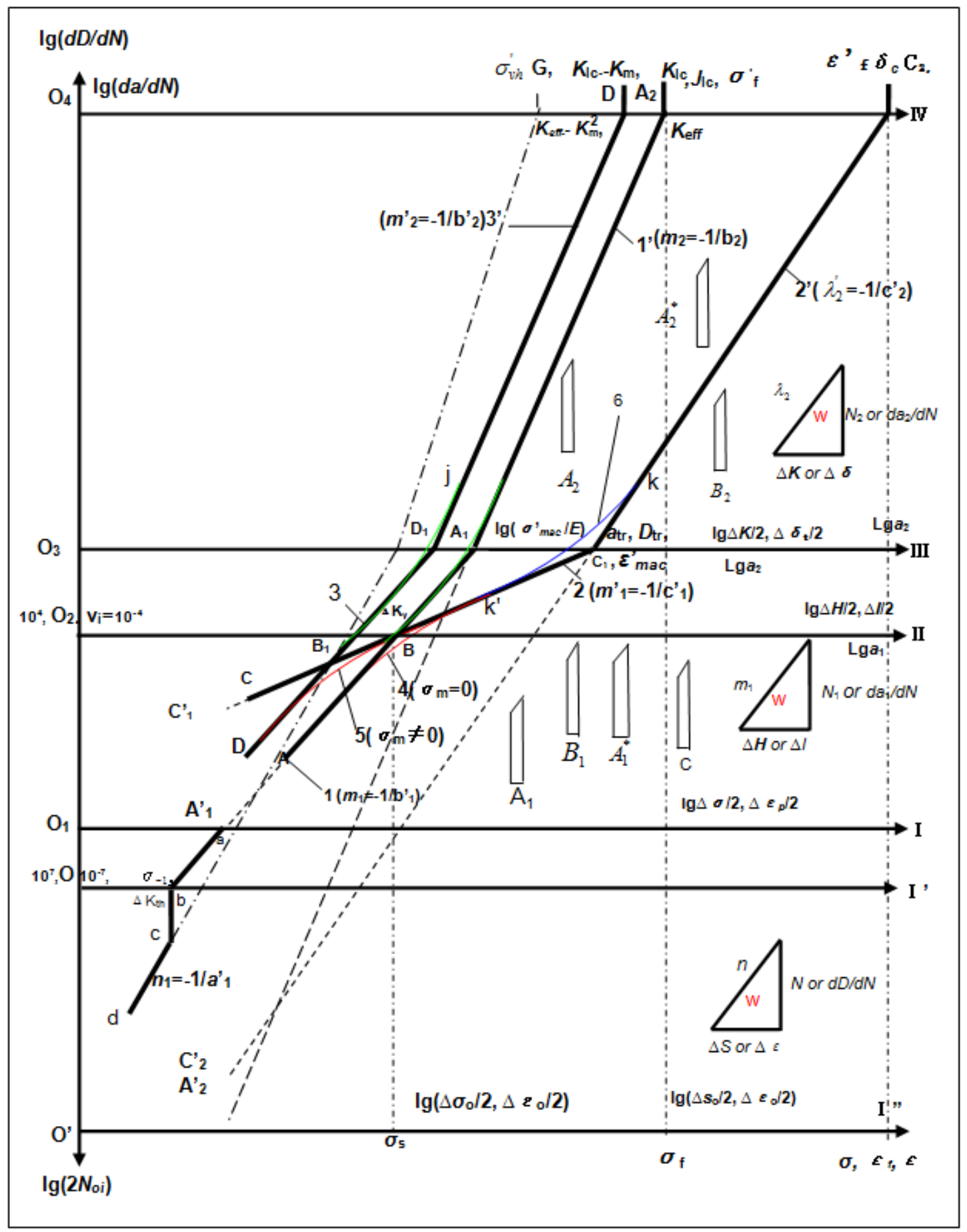

Figure 1. Calculating figure of material behaviors 1 (Or called Comprehensive figure of material behaviors or Bidirectional combined coordinate system and simplified schematic curves in the whole process) [1-3].

About life curves of short crack growth as the first stage are just described with curves $1\left(\sigma<\sigma_{s}, \sigma_{m}=0\right), 2\left(\sigma>\sigma_{s}\right)$ and $\left.3\left(\sigma<\sigma_{s}, \sigma_{m} \neq 0\right)\right)$ in reversed direction coordinate system inside attach figure.1, where depicting for the 
coordinate system constituting and each curve meaning had explained in references [1-3].

(1) Under work tress $\sigma<\sigma_{s}$ (high cycle fatigue) condition

Under work tress $\sigma<\sigma_{s}$ condition, it is with $a_{1}$ as variable to adopt the simple stress parameter $\sigma$ as its calculating one, and with the short crack-stress-factor range $\Delta H$ to express the life prediction equation, that corresponding reversed curves 1 and 3 can be calculated by following equations

$$
N_{1}=\int_{a_{01}}^{a_{t r}} \frac{d a_{1}}{A_{1} \times\left(\Delta H_{1}\right)^{m_{1}}},(\text { cycle })
$$

or

$$
N_{1}=\int_{a_{01}}^{a_{t r}} \frac{d a_{1}}{A_{1} \times(\Delta \sigma)^{m_{1}} a_{1}},(\text { cycle })
$$

Where

$$
\begin{array}{r}
H_{1}=\sigma \cdot a_{1}^{1 / m_{1}},\left(M P a \cdot m^{1 / m_{1}}\right) \\
\Delta H_{1}=\Delta \sigma \cdot a_{1}^{1 / m_{1}}\left(M P a \cdot m^{1 / m_{1}}\right)
\end{array}
$$

Here the $H$ in eqn (2) is defined as short crack stress intensity factor, it is driving force of short crack growth under monotonic loading, and the crack stress intensity factor range $\Delta H_{1}$ in eqn (3) it is driving force under fatigue loading. The $A_{1}$ is defined as the comprehensive and calculable material constant. Author researches and thinks, its physical meaning of the $A_{1}$ is a concept of power, that just is a maximal increment value to give out energy in one cycle before to cause material failure. Its geometrical meaning is a maximal micro-trapezium area approximating to beeline (Fig1), that is a projection of corresponding to curve $1\left(\sigma_{m}=0\right)$ or 3 $\sigma_{m} \neq 0$ on the y-axis, also is an intercept between $O_{1}-O_{3}$. Its slope of micro-trapezium bevel edge just is corresponding to the exponent $m_{1}$ of the formula (4-5).The comprehensive material constant $A_{1}$ in formulas (4-5) is a calculable one, it has function relation with other material constants $m_{1}$ and $\sigma_{f}^{\prime}$, etc. the $\sigma_{f}^{\prime}$ is a fatigue strength coefficient.

$$
\begin{gathered}
A_{1}=2\left(2 \sigma_{f}^{\prime}\right)^{-m_{1}} \times\left(v_{e f f}\right)^{-1},\left(M P a^{m_{1}} \mathrm{~mm} / \text { cycle }\right),\left(\sigma_{m}=0\right) \\
A_{1}=2\left[2 \sigma_{f}^{\prime}\left(1-\sigma_{m} / \sigma_{f}^{\prime}\right)\right]^{-m_{1}} \times\left(v_{e f f}\right)^{-1},\left(M P a^{m_{1}} \mathrm{~mm} / \text { cycle }\right), \\
\left(\sigma_{m} \neq 0\right)
\end{gathered}
$$

Here should yet explain the $A_{1}$ in eqn (4) is corresponding reversed curves 1 , its mean stress $\sigma_{m}=0$; The $A_{1}$ in eqn (5) is corresponding curves 3 , its $\sigma_{m} \neq 0$. And the correctional method for its mean stress $\sigma_{m} \neq 0$ can be corrected by reference [9].

Where

$$
\left.v_{e f f}=\ln \left(a_{1 f} / a_{0}\right) / N_{1 f c}-N_{01}=\left[\ln \left(a_{1 f} / a_{0}\right)-\ln a_{1} / a_{01}\right)\right] / N_{1 f}-N_{01},(m m / \text { cycle })
$$

or

$$
v_{e f f}=\left[a_{1 f} \ln (1 / 1-\psi)\right] / N_{1 f}-N_{01},(\mathrm{~mm} / \text { cycle })
$$

The $v_{\text {eff }}$ in eqns (6-7) is defined as an effective damage history correction factor in first stage, its physical meaning is the effective crack growth rate of whole failure to cause specimen material in a cycle, its unit is $\mathrm{mm} /$ cycle. $\psi$ is a reduction of area. $a_{0}$ is pre-micro-crack value that is no effect to fatigue damage under prior cycle loading [10]. $a_{01}$ is an initial miro-crack value, $a_{f}$ is a critical fracture size before failure. $N_{01}$ is initial life in first stage, $N_{01}=0 ; N_{1 f}$ is failure life, $N_{1 f}=1$. By the way, here is also to adopt those material constants $\sigma_{f}^{\prime}, b_{1}^{\prime}, \varepsilon_{f}^{\prime}, c_{1}^{\prime}$ as "genes" in the fatigue damage subject.

So, for the eqn (1), its final expansion equation corresponded reversed to curves $1\left(A_{1} A\right)$ is as below form:

$$
N_{1}=\frac{\ln a_{t r}-\ln a_{1}}{2\left(2 \sigma_{f}^{\prime}\right)^{-m_{1}} \times\left(v_{e f f}\right)^{-1}(\Delta \sigma)^{m_{1}}},(\text { Cycle }),\left(\sigma<\sigma_{s}, \sigma_{m}=0\right)
$$

And its final expansion equation corresponded reversed to curves $3\left(D_{1} D\right)$ should be:

$$
N_{o i}=\frac{\ln a_{o i}-\ln a_{1}}{2\left[2 \sigma_{f}^{\prime}\left(1-\sigma_{m} / \sigma_{f}\right)\right]^{-m_{1}} \times\left(v_{e f f}\right)^{-1} \times(\Delta \sigma)^{m_{1}}}\left(\sigma_{m} \neq 0\right)
$$

Where $a_{t r}$ is a transitional crack size from short crack to long crack growth process, $a_{t r} \approx a_{\text {mac }}, a_{\text {mac }}$ is a long crack size corresponding forming macro crack. $a_{o i}$ is a medial crack size between initial crack size and transitional crack size corresponding medial life $N_{o i}$.

(2) Under work stress $\sigma>\sigma_{s}$ (low cycle fatigue) condition. Under $\sigma>\sigma_{s}$ condition, here it is still with $a_{1}$ as variable, but it should adopt the simple strain $\varepsilon_{p}$ as its calculating parameter, and it should adopt the short crack-strain-factor range $\Delta I$ to express its life equation. That is corresponded to reversed direction curve $C_{1} C$ in Fig1, is as following form

$$
N_{1}=\int_{a_{1}}^{a_{t r}} \frac{d a_{1}}{B_{1} \times(\Delta I)^{m_{1}^{\prime}}},(\text { Cycle }),\left(\sigma>\sigma_{s}\right)
$$




$$
\begin{gathered}
\Delta I_{1}=\left(\Delta \varepsilon_{p}\right)^{m_{1}^{\prime}} \cdot a_{1} \\
N_{1}=\int_{a_{1}}^{a_{t r}} \frac{d a_{1}}{B_{1} \times\left(\Delta \varepsilon_{p}\right)^{m_{1}^{\prime}} a_{1}},(\text { Cycle }),\left(\sigma>\sigma_{s}\right)
\end{gathered}
$$

Where the $B_{1}$ is also calculable comprehensive material constants.

$$
B_{1}=2\left[2 \varepsilon_{f}^{\prime}\right]^{-m_{1}^{\prime}} \times\left(v_{e f f}\right)^{-1},(\%)^{m_{1}^{\prime}}(m m / \text { cycle })
$$

If via the crack stress factor amplitude $\Delta H_{1} / 2$ in eqn (10) to express it, due to plastic strain occur cyclic hysteresis loop effect, it should be

$$
N_{1}=\int_{a_{1}}^{a_{t r}} \frac{d a_{1}}{A_{1} \times(\Delta \sigma / 2)^{m_{1}} \times a_{1}},(\text { Cycle }),\left(\sigma>\sigma_{s}\right)
$$

Where the $A_{1}$ is also a calculable comprehensive material constant:

$$
A_{1}=2\left(2 \sigma_{f}^{\prime}\right)^{-m_{1}}\left(v_{\text {eff }}\right)^{-1},(M P a)^{m_{1}} \mathrm{~mm} / \operatorname{cycle}\left(\sigma_{m}=0\right)
$$

Or

$$
A_{1}=2 K^{\prime-m_{1}}\left[2 \varepsilon_{f}^{\prime}\left(1-\sigma_{m} / \sigma_{f}\right)\right]^{1 / c^{\prime}} \times\left(v_{e f f}\right)^{-1},(M P a)^{m_{1}} m m / \text { cycle }\left(\sigma_{m} \neq 0\right)
$$

Where $K^{\prime}$ is a cyclic strength coefficient. $m_{1}^{\prime}$ is defined to be fatigue ductility exponent, $m_{1}^{\prime}=-1 / c_{1}^{\prime}$, $m_{1}=-1 / c_{1}^{\prime} \times n^{\prime}, c_{1}^{\prime}$ just is a fatigue ductility exponent under low cycle fatigue, $n^{\prime}=b_{1}^{\prime} / c_{1}^{\prime}, n^{\prime}$ is a strain hardening exponent. So that, its final expansion equation for (10) is as below form,

$$
N_{o i}=\frac{\ln a_{o i}-\ln a_{1}}{2\left[2 \sigma_{f}^{\prime}\left(1-\sigma_{m} / \sigma_{f}\right)\right]^{-m_{1}}\left(v_{e f f}\right)^{-1} \times(\Delta \sigma / 2)^{m_{1}}}\left(\sigma>\sigma_{s}, \sigma_{m} \neq 0\right)
$$

If to take formula (17) to replace $A_{1}$ into eqn. (14), its final expansion equation is as below forming

$$
N_{o i}=\frac{\ln a_{o i}-\ln a_{1}}{2 K^{\prime-m_{1}}\left[2 \varepsilon^{\prime}{ }_{f}\left(1-\sigma_{m} / \sigma_{f}\right)\right]^{1 / c^{\prime}} \times\left(v_{e f f}\right)^{-1} \times(\Delta \sigma / 2)^{m_{1}}}\left(\sigma>\sigma_{s}, \sigma_{m} \neq 0\right)
$$

Here influence of mean stress in eqn (19-20) can be ignored.

\subsection{The Calculations for Fatigue-Damage in Long Crack \\ Growth Process (or Called the Second Stage)}

In Fig.1, the residual life curves of long crack growth in the second stage are just described with curves 1' $\left(\sigma<\sigma_{s}, \sigma_{m}=0\right), 2^{\prime}\left(\sigma>\sigma_{s}\right)$ and $\left.3^{\prime}\left(\sigma<\sigma_{s}, \sigma_{m} \neq 0\right)\right)$ at reversed direction coordinate system.

(1) Under work tress $\sigma<\sigma_{s}$ condition

Here it is divided two methods: $K_{2}$-factor method and $\sigma$ -method:

1) $K_{2}$-factor method

For life prediction equation corresponded to reversed curves $A_{2} A_{1}$ and $D_{2} D_{1}$ should be as following

$$
N_{2}=\int_{a_{t r}}^{a_{\text {eff }}} \frac{d a_{2}}{A_{2} \times\left[y_{2}(a / b) \Delta K_{2}\right]^{m_{2}}},(\text { cycle })
$$

Where

$$
\begin{gathered}
K_{2}=\sigma \sqrt{\pi a_{2}} \\
\Delta K_{2}=\Delta \sigma \sqrt{\pi a_{2}}
\end{gathered}
$$

As it is known the $K_{2}=K_{\mathrm{I}}$-factor is just the macro crack stress intensity factor, and the $\Delta K_{2}$ is the macro-crack stress intensity factor range. The $y_{2}(a / b)$ is correction factor [11] related for long crack form and structure size. The $A_{2}$ in eqn.(21) is defined as comprehensive material constants of macro-crack, for $\sigma_{m}=0$, it is corresponding curve $A_{1} A_{2}$, and it is also calculable one as following

$A_{2}=2\left(2 K_{2 e f f}\right)^{-m_{2}} \times v_{p v},(M P a \sqrt{m})^{m_{1}} \times m m /$ cycle $),\left(\sigma_{m}=0\right)$

Or 


$$
\left.A_{2}=\frac{\frac{2}{2-m_{2}}\left(a_{2 e f f}^{1-\frac{m_{2}}{2}}-a_{02}^{1-\frac{m_{2}}{2}}\right)}{\left(2 \sigma_{2 e f f} \sqrt{\pi}\right)^{m_{2}}\left(N_{2 e f f}-N_{02}\right)},(M P a \sqrt{m})^{m_{1}} \times m m / c y c l e\right),\left(\sigma_{m}=0\right)
$$

For $\sigma_{m} \neq 0$, the $A_{2}$ is corresponded to curve $D_{1} D_{2}$, it should be as following form

$$
A_{2}=2\left[2 K_{2 e f f}\left(1-K_{2 m} / K_{2 f c}\right)\right]^{-m_{2}} \times v_{p v},\left(\sigma_{m} \neq 0\right)
$$

Where $K_{2 m}$ is mean crack stress intensity factor, $K_{2 e f f}$ is an effective crack stress intensity factor, $K_{2 f c}$ is a critical crack stress intensity factor, which they are parameters under cyclic loading. It should be point that the physical meaning for the $A_{2}$ is also a concept of power, that just is a maximal increment value to give out energy in one cycle before failure. Its geometrical meaning is also a maximal micro-trapezium area approximating to beeline (Fig1), that is a projection of corresponding to curve 1' $\left(\sigma_{m}=0\right)$ or $3^{\prime} \sigma_{m} \neq 0$ on the y-axis, also is an intercept between $O_{3}-O_{4}$. Its slope of micro-trapezium bevel edge just is corresponding to the exponent $m_{2}$ of the formula (24 26). Here,

$$
v_{p v}=\frac{\left(a_{2 p v}-a_{02}\right)}{N_{2 e f f}-N_{02}} \approx 3 \times 10^{-5} \sim 3 \times 10^{-4}=v^{*}(\mathrm{~mm} / \text { Cycle })
$$

Author research and think, the parameter $v_{p v}$ is defined to be the virtual rate, it is an equivalent rate caused in precrack, it can take similar dimension with the " $v^{*}$ " $(\mathrm{m} /$ cycle $)$ by reference [12]. But its unit is different, here unit of the $v_{p v}$ is " $\mathrm{mm} / \mathrm{cycle}$ ". The crack size $a_{2 p v}$ is a virtual crack size as equivalent to a precrack size, $a_{02}$ is an initial as equivalent to the initial micro-crack size. $N_{02}$ is an initial life, $N_{02}=0$. $N_{p v}$ is a virtual life, $N_{2 e f f}=1$. In references [13-14], all refer to effective stress intensity factor, here to propose to take

$$
N_{2 e f f}=\frac{\frac{2}{2-m_{2}}\left(a_{2 e f f}^{1-\frac{m_{2}}{2}}-a_{02}^{1-\frac{m_{2}}{2}}\right)}{2\left[2 K_{2 e f f}\left(1-K_{m} / K_{2 f c}\right)\right]^{-m_{2}} \times v_{p v} \times\left[(Y a / b)^{m_{2}} \Delta \sigma^{m_{2}} \pi^{\frac{m_{2}}{2}}\right]},\left(\sigma_{m} \neq 0\right)
$$

Its medial life $N_{2 o j}$ in second stage is

$$
N_{2 o j}=\frac{\frac{2}{2-m_{2}}\left(a_{o j}^{1-\frac{m_{2}}{2}}-a_{t r}^{1-\frac{m_{2}}{2}}\right)}{2\left[2 K_{2 e f f}\left(1-K_{2 m} / K_{2 f c}\right)\right]^{-m_{1}} \times v_{p v}\left[y_{2}(a / b) \Delta \sigma \sqrt{\pi}\right]^{m_{2}}},(\operatorname{cycle})\left(\sigma_{m} \neq 0\right)
$$

Where $a_{t r}$ is a transitional crack size between two stages from short crack $a_{\text {mic }}$ to long crack $a_{\text {mac }}$ growth process, $a_{t r} \approx a_{m a c}$, the $a_{o j}$ is a medial size. $a_{o 2}<a_{o j}<a_{2 e f f}$.

2) $\sigma$-method

Due to word stress is still $\sigma / \sigma_{s}<<1\left(\sigma \leq 0.5 \sigma_{s}\right)$, in the long crack growth process, its residual life equation of corresponding reversed direction curve $A_{2} A_{1}$ and $D_{2} D_{1}$ in fig. 1 is as following form

$$
N_{1}=\int_{a_{t r}}^{a_{2 e f f}} \frac{d a_{1}}{B_{2} \times\left(\Delta \delta_{t}\right)^{m_{2}}},(\text { Cycle })
$$


Where

$$
\begin{gathered}
\delta_{t}=\pi a_{2} \sigma_{s} \times\left(\sigma / \sigma_{s}\right)^{2} / E,(\mathrm{~mm}) \\
\Delta \delta_{t}=\frac{\beta \Delta \sigma^{2} \pi a_{2}}{4 \sigma_{s} E},(\mathrm{~mm}),\left(\sigma_{m}=0\right)
\end{gathered}
$$

The $\delta_{t}$ is a crack tip open displacement, it is driving force of short crack growth under monotonic loading; and the $\Delta \delta_{t}$ is a crack tip open displacement range, it is driving force under fatigue loading. For the coefficient $\beta$ in eqn (39), it equal
1( $\beta=1)$ under plane stress condition, under plane strain condition, $\beta=\left(1-v^{2}\right) / 2 . v$ is Poisson's ratio. $E$ is an elasticity modulus. The $B_{2}$ is a comprehensive material constant in second stage, its physical meaning of the $B_{2}$ is also a concept of power. Its geometrical meaning is also a maximal micro-trapezium area approximating to beeline (Fig1). $B_{2}$ is also calculable comprehensive material constant, for $\sigma=0$ that is

$$
B_{2}=2\left(\frac{\beta\left(\sigma_{f}^{\prime 2} \times a_{2 e f f} \times \pi / \sigma_{s}^{2}\right) \sigma_{s}}{E}\right)^{-m_{2}} \times v_{p v},\left(m m^{m_{2}} \times m m / \text { cycle }\right), \quad(\sigma=0)
$$

And for $\sigma \neq 0$ that is

$$
B_{2}=2\left(\frac{2 \beta\left(\sigma_{f}^{\prime 2} \times a_{2 e f f} \times \pi / \sigma_{s}^{2}\right) \sigma_{s}}{E}\left[1-\frac{a_{02}\left(\sigma_{\max }^{2}+\sigma_{\min }^{2}\right)}{2 \times a_{2 e f f} \sigma_{s}^{2}}\right]\right)^{-m_{2}} v_{p v},\left(m m^{m_{2}} \times m m / \text { cycle }\right) \quad(\sigma \neq 0)
$$

Where $\sigma_{\max }$ and $\sigma_{\min }$ are maximum and minimum work curve $A_{2} A_{1}$ for eqn (37) is as below, stress. The $a_{2 e f f}$ can be calculable effective crack size.

So its final expansion form corresponded reversed direction

$$
N_{2 e f f}=\frac{\left(4 E \cdot \sigma_{s}\right)^{m_{2}} \times \frac{1}{1-m_{2}}\left(a_{2 e f f}{ }^{1-m_{2}}-a_{t r}{ }^{1-m_{2}}\right)}{2\left(\frac{2 \beta\left(\sigma_{2 e f f}^{2} \times a_{2 e f f} \times \pi / \sigma_{s}^{2}\right) \sigma_{s}}{E}\right)^{-m_{2}} v_{p v}\left[y_{2}(a / b) \beta \times \Delta \sigma^{2} \pi\right]^{m_{2}}},\left(\sigma_{m}=0\right),(\text { cycle })
$$

And the life equation corresponded to reversed direction curve $D_{2} D_{1}$ is following

$$
N_{2 e f f}=\frac{\left(2 E \cdot \sigma_{s}\right)^{m_{2}} \times \frac{1}{1-m_{2}}\left(a_{2 e f f}{ }^{1-m_{2}}-a_{t r}{ }^{1-m_{2}}\right)}{2\left(\frac{2 \beta\left(\sigma_{2 e f f}^{2} \times a_{2 e f f} \times \pi / \sigma_{s}^{2}\right) \sigma_{s}}{E}\left[1-\frac{a_{02}\left(\sigma_{\max }^{2}+\sigma_{\min }^{2}\right)}{2 \times a_{\text {meff }} \times \sigma_{s}^{2}}\right]\right)^{-m_{2}} v_{p v}^{\prime}\left[y_{2}(a / b) \beta \Delta \sigma^{2} \pi\right]^{m_{2}}},(\text { cycle })\left(\sigma_{m} \neq 0\right)
$$

(2) Under work tress $\sigma>\sigma_{s}$ condition

Under $\sigma>\sigma_{s}$ condition, its effective life models corresponded to reversed curve $C_{2} C_{1}$ in figure 1 is as below form,

$$
N_{2 e f f}=\int_{a_{t r}}^{a_{2 e f f}} \frac{d a_{2}}{B_{2} \times\left(\Delta \delta_{t} / 2\right)^{\lambda_{2}}},(\text { Cycle }),\left(\sigma>\sigma_{s}\right)
$$

Where $B_{2}$ is also a calculable comprehensive material constant,

$$
\begin{gathered}
B_{2}=2\left[\left(\pi \sigma_{s}\left(\sigma_{f}^{\prime} / \sigma_{s}+1\right) a_{2 e f f} / E\right)\right]^{-\lambda_{2}} \times v_{p v},\left(m^{\lambda_{2}} \times m m / \text { cycle }\right),\left(\sigma_{m}=0\right) \\
B_{2}=2\left[\left(\pi \sigma_{s}\left(\sigma_{f}^{\prime} / \sigma_{s}+1\right)\left(1-\sigma_{m} / \sigma_{f}^{\prime}\right) a_{2 e f f} / E\right)\right]^{-\lambda_{2}} \times v_{p v},\left(m^{\lambda_{2}} \times m m / c y c l e\right),\left(\sigma_{m} \neq 0\right)
\end{gathered}
$$

The $\lambda_{2}$ is defined to be ductility exponent in long crack growth process, $\lambda_{2}=-1 / c_{2}^{\prime}, c_{2}^{\prime}$ is a fatigue ductility exponent in second stage.
So that, the final expansion equations is derived from above mentioned eqn. (44) as follow

For $\sigma_{m}=0$, 


$$
N_{2 e f f}=\frac{\frac{1}{1-\lambda_{2}}\left(a_{2 e f f}{ }^{1-\lambda_{2}}-a_{02}{ }^{1-\lambda_{2}}\right)}{2\left[\left(\pi \sigma_{s}\left(\sigma_{f}^{\prime} / \sigma_{s}+1\right) a_{2 e f f} / E\right)\right]^{-\lambda_{2}} \times v_{p v}\left[\frac{0.5 \pi \sigma_{s} y_{2}(a / b)\left(\Delta \sigma / 2 \sigma_{s}+1\right)}{E}\right]^{\lambda_{2}}},(\text { cycle }),
$$

For $\sigma_{m} \neq 0$, it should be

$$
N_{2 e f f}=\frac{\frac{1}{1-\lambda_{2}}\left(a_{2 e f f}{ }^{1-\lambda_{2}}-a_{02}{ }^{1-\lambda_{2}}\right)}{2\left[\left(\pi \sigma_{s}\left(\sigma_{f}^{\prime} / \sigma_{s}+1\right)\left(1-\sigma_{m} / \sigma_{f}^{\prime}\right) a_{2 e f f} / E\right)\right]^{-\lambda_{2}} \times v_{p v}\left[\frac{0.5 \pi \sigma_{s} y_{2}\left(\Delta \sigma / 2 \sigma_{s}+1\right)}{E}\right]^{\lambda_{2}}},(\text { cycle })
$$

In the eqn (48), influence to mean stress usually can ignore. Where, the $a_{2 e f f}$ is an effective crack size, it can calculate from effective crack tip opening displacement $\delta_{2 e f f}$

$$
a_{2 e f f}=\frac{E \times \delta_{2 e f f}}{\pi \sigma_{s}\left(\sigma_{f}^{\prime} / \sigma_{s}+1\right)},(\mathrm{mm})
$$

And

$$
\delta_{2 e f f}=(0.25 \sim 0.4) \delta_{c},(\mathrm{~mm})
$$

Where the $\delta_{c}$ is critical crack tip displacement. So the $a_{2 \text { eff }}$ in (47-48) can be calculated by $\delta_{c}$-value by means of equations (49-50).

\subsection{The Life Prediction Calculations for Crack Propagation in Whole Process}

$$
\begin{aligned}
& \frac{d a_{1}}{d N}=\left\{2\left[2 \sigma_{f}^{\prime}\right]^{-m_{1}} \times\left(a_{f} \cdot v_{e f f}\right)^{-1} \times(\Delta \sigma)^{m_{1}} a\right\}_{a_{01} \rightarrow a_{t r}}<=\frac{d a_{t r}}{d N}= \\
& =<\frac{d a_{2}}{d N_{2}}=\left\{2\left(\frac{2 \beta\left(\sigma^{2}{ }_{2 e f f} \times a_{e f f} \pi / \sigma^{2}{ }_{s}\right) \sigma_{s}}{E}\right)^{-m_{2}} v_{p v} \times\left(\frac{y_{2}(a / b) \beta \Delta \sigma^{2} \pi a}{4 \sigma_{s} E}\right)^{m_{2}}\right\}_{a_{t r} \rightarrow a_{e f f}},\left(\sigma_{m}=0\right),(m m / \text { cycle })
\end{aligned}
$$

For $\sigma<\sigma_{s}, \sigma_{m} \neq 0$, its expanded the crack curve $D D_{1} D_{2}$ is as following form rate-linking-equation for eqn (51) corresponded to positive

$$
\begin{aligned}
& \frac{d a_{1}}{d N}=\left\{2\left[2 \sigma_{f}^{\prime}\left(1-\sigma_{m} / \sigma_{f}^{\prime}\right)\right]^{-m_{1}} \times\left(a_{f} \cdot v_{e f f}\right)^{-1} \times(\Delta \sigma)^{m_{1}} a\right\}_{a_{01} \rightarrow a_{t r}}<=\frac{d a_{t r}}{d N}= \\
& =<\frac{d a_{2}}{d N_{2}}=\left\{2\left(\frac{2 \beta\left(\sigma_{e f f}^{2} \times a_{e f f} \times \pi / \sigma_{s}^{2}\right) \sigma_{s}}{E}\left[1-\frac{a_{02}\left(\sigma_{\max }^{2}+\sigma_{\min }^{2}\right)}{2 a_{\text {meff }} \sigma_{s}^{2}}\right]\right)^{-m_{2}} v_{p v} \times\left(\frac{y_{2}(a / b) \beta \Delta \sigma^{2} \pi a}{2 \sigma_{s} E}\right)^{m_{2}}\right\}_{a_{t r} \rightarrow a_{e f f}},
\end{aligned}
$$$$
m m / \operatorname{cycle},\left(\sigma_{m} \neq 0\right)
$$

And the life equations in whole process corresponding to reversed direction curves $A_{2} A_{1} A$ and $D_{2} D_{1} D$ should be as below

$$
\sum N=N_{1}+N_{2}=\int_{a_{01}}^{a_{t r}} \frac{d a}{A_{1} \times(\Delta \sigma)^{m_{1}} \times a}+\int_{a_{t r}}^{a_{2 e f f}} \frac{d a}{A_{2}\left(\Delta \delta_{t}\right)^{m_{2}}},
$$

Its expanded equation corresponding to reversed direction curves $A_{2} A_{1} A$ is as following form 


$$
\begin{aligned}
& \sum N=N_{1}+N_{2}=\int_{a_{01}}^{a_{t r}} \frac{d a}{2\left[2 \sigma_{f}^{\prime}\right]^{-m_{1}} \times\left(a_{f} \cdot v_{e f f}\right)^{-1} \times(\Delta \sigma)^{m_{1}} \times a} \\
& +\int_{a_{t r}}^{a_{2 e f f}} \frac{d a}{\left.2\left(\frac{2 \beta\left(\sigma_{e f f}^{2} \times \pi \times a_{e f f} / \sigma_{s}^{2}\right) \sigma_{s}}{E}\right)^{-m_{2}} v_{p v}\left[2 y_{2} \beta \Delta \sigma^{2} \pi a\right) / 4 E \sigma_{s}\right]^{m_{2}}},(\text { cycle }),\left(\sigma_{m}=0\right)
\end{aligned}
$$

But for $\sigma_{m} \neq 0$, its expanded equation corresponding to reversed direction curves $D_{2} D_{1} D$ should be

$$
\begin{aligned}
& \sum N=N_{1}+N_{2}=\int_{a_{01}}^{a_{t r}} \frac{d a}{2\left[2 \sigma_{f}^{\prime}\left(1-\sigma_{m} / \sigma_{f}^{\prime}\right)\right]^{-m_{1}} \times\left(a_{f} \cdot v_{e f f}\right)^{-1} \times(\Delta \sigma)^{m_{1}} \times a} \\
& +\int_{a_{t r}}^{a_{\text {eeff }}} \frac{d a}{\left.2\left(\frac{2 \beta\left(\sigma_{e f f}^{2} \times a_{e f f} \pi / \sigma_{s}^{2}\right) \sigma_{s}}{E}\left[1-\frac{a_{02}\left(\sigma_{\max }^{2}+\sigma_{\min }^{2}\right)}{2 a_{\text {meff }} \sigma_{s}^{2}}\right]\right)^{-m_{2}} v_{p v}\left[2 y_{2} \beta \Delta \sigma^{2} \pi a\right) / 2 E \sigma_{s}\right]^{m_{2}}},(\text { cycle })\left(\sigma_{m} \neq 0\right)
\end{aligned}
$$

(2) Under work stress $\sigma>\sigma_{s}$

Under work stress $\sigma>\sigma_{s}$, its expanded rate link equation

$$
\begin{aligned}
& \frac{d a_{1}}{d N}=\left\{2 K^{\prime-m_{1}}\left[2 \varepsilon^{\prime}{ }_{f}\right]^{1 / c^{\prime}} \times\left(v_{f} \times a_{t r}\right)^{-1} \times(\Delta \sigma / 2)^{m_{1}} \times a\right\}_{a_{01} \rightarrow a_{t r}}<=\frac{d a_{t r}}{d N}= \\
& \frac{d a_{2}}{d N_{2}}=<\left\{2\left[\left(\pi \sigma_{s}\left(\sigma_{f}^{\prime} / \sigma_{s}+1\right) a_{e f f} / E\right)\right]^{-\lambda_{2}} \times v_{p v}\left[\frac{0.5 \pi \sigma_{s} y_{2}\left(\Delta \sigma / 2 \sigma_{s}+1\right) a}{E}\right]^{\lambda_{2}}\right\}_{a_{t r} \rightarrow a_{e f f}}, m m / c y c l e,(\sigma \neq 0)
\end{aligned}
$$

The life equations in whole process corresponding to reversed direction curve $C_{2} C_{1} C$ should be as following

$$
\sum N=N_{1}+N_{2}=\int_{a_{01}}^{a_{t r}} \frac{d a}{B_{1} \times(\Delta \sigma / 2)^{m_{1}} \times a}+\int_{a_{t r}}^{a_{2 e f f}} \frac{d a}{B_{2}\left(\Delta \delta_{t} / 2\right)^{\lambda_{2}}},
$$

And the life prediction expanded expression in whole process corresponded reversed curve $C_{2} C_{1} C$, it should be

$$
\begin{aligned}
& \sum N=\int_{a_{01}}^{a_{t r}} \frac{d a}{2 K^{1-m_{1}}\left[2 \varepsilon_{f}^{\prime}\right]^{1 / c^{\prime}} \times\left(D_{f} \cdot v_{e f f}\right)^{-1} \times(\Delta \sigma / 2)^{m_{1}} \times a} \\
& +\int_{a_{t r}}^{a_{2 e f f}} \frac{d a}{2\left[\left(\pi \sigma_{s}\left(\sigma_{f}^{\prime} / \sigma_{s}+1\right) a_{2 e f f} / E\right)\right]^{-\lambda_{2}} \times v_{p v}\left[\frac{0.5 \pi \sigma_{s} y_{2}\left(\Delta \sigma / 2 \sigma_{s}+1\right) a}{E}\right]^{\lambda_{2}}},(\text { cycle })
\end{aligned}
$$

It should point that the calculations for rate and life in whole process should be according to different stress level, to select appropriate calculable equation. Here it have to explain that its meaning of the crack rate-linking-equation $(51-53,57)$ is to make a calculable linking formula between the first stage crack rate and the second stage one, which it should be calculated by the short crack growth rate equation before the transition point size $a_{t r}$; it should be calculated by the long crack growth rate equation after the transition point $a_{t r}$, note that it is not added together by the crack growth rates for two stages. But the life calculations for two stages can be added together. About calculation method, it can calculate by means of computer doing computing by different crack size [16].

\section{Calculating Example}

\subsection{Contents of Example Calculations}

To suppose a pressure vessel is made with elastic-plastic steel $16 \mathrm{MnR}$, its strength limit of material $\sigma_{b}=573 \mathrm{MPa}$, yield limit $\sigma_{s}=361 \mathrm{MPa}$, fatigue limit $\sigma_{-1}=267.2 \mathrm{MPa}$, reduction of area is $\psi=0.51$, modulus of elasticity $E=200000 \mathrm{MPa}$; Cyclic strength coefficient $K^{\prime}=1165 \mathrm{MPa}$, strain-hardening exponent $n^{\prime}=0.187$; Fatigue strength coefficient $\sigma_{f}^{\prime}=947.1 \mathrm{MPa}$, fatigue strength exponent $b_{1}^{\prime}=-0.111, \quad m_{1}=9.009 ;$ Fatigue ductility coefficient $\varepsilon^{\prime}{ }_{f}=0.464$, fatigue ductility exponent $c_{1}^{\prime}=-0.5395$, 
$m_{1}^{\prime}=1.8536$. Threshold value $\Delta K_{t h}=8.6 \mathrm{MPa} \sqrt{\mathrm{m}}$, critical stress intensity factor $K_{2 c}=K_{1 c}=92.7 \mathrm{MPa} \sqrt{\mathrm{m}}$. Working stress $\sigma_{\max }=450 \mathrm{MPa}, \sigma_{\min }=0$ in pressure vessel. And suppose that for long crack shape has been simplified via treatment become an equivalent through-crack, the correction coefficient $y_{2}(a / b)$ of crack shapes and sizes equal 1 , i.e. $y_{2}(a / b)=1$. Other computing data are all included in table 1 .

Table 1. Computing data.

\begin{tabular}{lllllllll}
\hline$K_{1 c}, M P a \sqrt{m}$ & $K_{e f f}, M P a \sqrt{m}$ & $K_{t h}, M P a \sqrt{m}$ & $v_{p v}$ & $m_{2}$ & $\delta_{c}, m m$ & $\lambda_{2}$ & $y_{2}(a / b)$ & $a_{t h}, m m$ \\
\hline 92.7 & 28.23 & 8.6 & $2 \times 10^{-4}$ & 3.91 & 0.18 & 2.9 & 1.0 & 0.07 \\
\hline
\end{tabular}

\subsection{Required Calculating Data}

Try to calculate respectively as following different data and depicting their curves:

(1) To calculate the transitional point crack size $a_{t r}$ between two stages;

(2) To calculate the crack growth rate at transitional point ( at crack size $a_{t r}$ )

(3) To calculate the life $N_{1}$ in first stage from micro crack $a_{1}=0.02 \mathrm{~mm}$ growth to transitional point $a_{t r}$

(4) To calculate the life $N_{2}$ in second stage $N_{2}$ from transitional point $a_{t r}$ to long crack size $a_{2 \mathrm{eff}}=5 \mathrm{~mm}$;

(5) Calculating the whole service lifetime $\sum N$.

(6) Depicting their life curves in whole process.

\subsection{Calculating Processes and Methods}

The concrete calculation methods and processes are as follows

(1) Calculations for relevant parameters

1) Stress range and mean stress calculations:

Stress range: $\Delta \sigma=\sigma_{\max }-\sigma_{\min }=450-0=450(\mathrm{MPa})$

Mean stress:

$\sigma_{m}=\left(\sigma_{\max }+\sigma_{\min }\right) / 2=(450-0) / 2=225 \mathrm{MPa}$

2) According to formulas (7), calculation for correction coefficient $v_{\text {eff }}$ in first stage.

For the effective crack sizes $a_{\text {eff }}$ in first stage and the second stage, it both can be calculated respectively, and can take smaller one of both, here to take same value with the second stage, $a_{1 e f f}=a_{2 e f f}=2 \mathrm{~mm}$. For example, according to formulas (49), Calculating effective size $a_{e f f}$

$a_{e f f}=\frac{E \times \delta_{e f f}}{\pi \sigma_{s}\left(\sigma_{f} / \sigma_{s}+1\right)}=\frac{200000 \times 0.25 \times 0.18}{\pi 361(947.1 / 361+1)}=2.1(\mathrm{~mm})$

Take $a_{\text {eff }}=2.0 \mathrm{~mm}$

$v_{\text {eff }}=a_{\text {eff }} \ln [1 /(1-\psi)]=2 \times \ln [1 /(1-0.51)]=1.43,(\mathrm{~mm} /$ cycle $)$

3 ) By eqn (27), to select virtual rate $v_{p v}$ in second stage, here take:

$v_{p v}=\frac{a_{2 e f f}-a_{02}}{N_{2 f}-N_{02}} \approx 2.0 \times 10^{-4}(\mathrm{~mm} /$ Cycle $), \quad N_{2 f}=1, \quad N_{02}=0$

(2) To calculate the crack size $a_{t r}$ of transitional point between two stages

1) To select calculating equation of short crack growth rate

At first, calculation for comprehensive material constant $B_{1}$ by eqn (17)

$$
\begin{aligned}
& A_{1}=2 K^{1^{-m_{1}}}\left[2{\varepsilon^{\prime}}_{f}\left(1-\sigma_{m} / \sigma_{f}^{\prime}\right)\right]^{1 / c^{\prime}} \times\left(a_{e f} \times v_{f}\right)^{-1}=2 \times 1165^{-9.01} \times[2 \times 0.464(1-225 / 947.1)]^{1 /-0.5395}(2 \times 0.713)^{-1} \\
& =6.28 \times 10^{-28},(M P a)^{m_{1}} \mathrm{~mm} / \mathrm{cycle}
\end{aligned}
$$

Here select the crack growth rate linking equation (57), and for its growth rate to simplify as follow form,

$$
d a_{1} / d N_{1}=A_{1} \times(\Delta \sigma / 2)^{m_{1}} \times a_{1}=3.193 \times 10^{-28} \times(450 / 2)^{9.01} \times a_{1}=6.28 \times 10^{-28} \times 1.56 \times 10^{21} \times a_{1}=9.8 \times 10^{-7} \times a_{1}
$$

2) By rate linking equation (57), calculating for crack growth rate in long crack growth process,

Calculation for comprehensive material constant $B_{2}$ by eqn (46)

$$
\begin{aligned}
& B_{2}=2\left[\left(\pi \sigma_{s}\left(\sigma_{f}^{\prime} / \sigma_{s}+1\right)\left(1-\sigma_{m} / \sigma_{f}^{\prime}\right) a_{e f f} / E\right)\right]^{-\lambda_{2}} \times v_{p v}=2[2(3.1416 \times 361(947.1 / 361+1)(1-225 / 947.1) \times 2 / 200000)]^{-2.9} \\
& \times 2 \times 10^{-4}=9.1988,(\mathrm{~mm})^{-\lambda_{2}} \times \mathrm{mm} / \text { Cycle }
\end{aligned}
$$

For the long crack rate in second stage, to take brief calculations as follow form, 


$$
\begin{aligned}
& d a_{2} / d N_{2}=B_{2}\left[\frac{0.5 \pi \sigma_{s} y_{2}\left(\Delta \sigma / 2 \sigma_{s}+1\right) a_{2}}{E}\right]^{\lambda_{2}}=9.1988 \times\left[\frac{0.5 \pi 361(450 /(2 \times 361)+1) a_{2}}{E}\right]^{2.9}=9.1988 \times 1.6698 \times 10^{-7} a_{2}^{2.9} \\
& =1.5384 \times 10^{-6} a_{2}^{2.9} \\
& \text { 3) Calculation for crack size } a_{t r} \text { at transitional point } \\
& \text { According to the equations (51) and (57), for calculation the } \quad a_{t r}=(0.638)^{\frac{1}{1.9}}=(0.638)^{0.5263}=0.789(\mathrm{~mm})
\end{aligned}
$$
crack size $a_{t r}$ at transitional point, it can make equal between both rate expansion equations, and to simplify it as following

$$
\begin{aligned}
& 6.28 \times 10^{-28} \times 1.56 \times 10^{21} \times a_{t r}=9.1988 \times 1.6698 \times 10^{-7} \times a_{t r}^{2.9} \\
& d a_{1} / d N_{1}=d a_{t r} / d N_{t r}=9.8 \times 10^{-7} a_{1}=9.8 \times 10^{-7} \times 0.789=7.74 \times 10^{-7}(\mathrm{~mm} / \mathrm{cycle}) \\
& d a_{2} / d N_{2}=d a_{t r} / d N_{t r}=1.5384 \times 10^{-6} a_{t r}^{2.9}=1.5384 \times 10^{-6} \times(0.79)^{2.9} \\
& =7.74 \times 10^{-7}(\mathrm{~mm} / \mathrm{cycle})
\end{aligned}
$$

Result, to obtain crack size $a_{t r}=0.789(\mathrm{~mm})$ at the transitional point.

(3) To calculate the crack growth rate at transitional point

Here it can be seen, the growth rate at the transition point is micro-crack $a_{01}=0.02 \mathrm{~mm}$ to transitional point same.

(4) Life prediction calculations in whole process

$$
a_{t r}=0.789 \mathrm{~mm} \text { is as follow, }
$$

1) To select eqn (20), to calculate the life $N_{1}$ from

$$
\begin{aligned}
& N_{1}=\frac{\ln a_{t r}-\ln a_{01}}{2 K^{1-m_{1}}\left[2 \varepsilon_{f}^{\prime}\left(1-\sigma_{m} / \sigma_{f}^{\prime}\right)\right]^{1 / c^{\prime}} \times\left(a_{e f f} \times v_{f}\right)^{-1} \times(\Delta \sigma / 2)^{m_{1}} \times a} \\
& =\frac{\ln 0.789-\ln 0.02}{2 \times 1165^{-9.01} \times[2 \times 0.464(1-225 / 947.1)]^{1 / 0.5395}(2 \times 0.713)^{-1} \times(450 / 2)^{9.01}} \\
& =\frac{3.675}{6.28 \times 10^{-28} \times 1.56 \times 10^{21}}=\frac{3.675}{9.8 \times 10^{-7}}=3751260(\text { Cycle })
\end{aligned}
$$

So predicting life in first stage $N_{1}=3751260$ (Cycle)

And for above formulas, we can derive simplified life equation corresponded to different crack size as follow form
2) To select eqn (48), to calculate the life $N_{2}$ from transitional point $a_{t r}=0.789 \mathrm{~mm}$ to $a_{2 e f f}=5 \mathrm{~mm}$ is as follow,

$$
\begin{aligned}
& N_{1}=\frac{1}{9.8 \times 10^{-7} a_{1}} \\
& N_{2}=\frac{\frac{1}{1-\lambda_{2}}\left(a_{2 e f f}{ }^{1-\lambda_{2}}-a_{t r}{ }^{1-\lambda_{2}}\right)}{2\left[\left(\pi \sigma_{s}\left(\sigma_{f}^{\prime} / \sigma_{s}+1\right)\left(1-\sigma_{m} / \sigma_{f}^{\prime}\right) a_{e f f} / E\right)\right]^{-\lambda_{2}} \times v_{p v}\left[\frac{0.5 \pi \sigma_{s} y_{2}\left(\Delta \sigma / 2 \sigma_{s}+1\right)}{E}\right]^{\lambda_{2}}} \\
& =\frac{\frac{1}{1-2.9}\left(5^{1-2.9}-0.789^{1-2.9}\right)}{2[(3.1416 \times 361(947.1 / 361+1)(1-225 / 947.1) \times 2 / 200000)]^{-2.9} \times v_{p v}} \\
& \times \frac{1}{\left[\frac{0.5 \pi 361(450 /(2 \times 361)+1)}{E}\right]^{2.9}}=\frac{0.8}{9.1988 \times 1.6698 \times 10^{-7}}=\frac{0.8}{1.5384 \times 10^{-6}}=520625 \text { (Cycle) }
\end{aligned}
$$

From above formulas, we can also derive simplified life equation corresponding different crack size as follow form

$$
N_{2}=\frac{1}{1.5384 \times 10^{-6} a_{2}^{2.9}}
$$

Therefore, predicting lifetime in whole process is

$$
\sum N=N_{1}+N_{2}=3751260+520625=4271885(\text { Cycle })
$$

The life data corresponded to different crack length is all included in table 2, 3 and 4 . Author finds these data are calculated by the simple stress- or strain-parameter method are basically close as compared with another result data that are calculated by the two-parameter-multiplication-method in damage mechanics, where it has been published recently in reference [3]. Especially, those dada in second stage are closer, and the calculating models and method is simpler than the two-parameter-method. 
Table 2. Crack growth life data in whole process.

\begin{tabular}{llllll}
\hline Data point of number & $\mathbf{1}$ & $\mathbf{2}$ & $\mathbf{3}$ & $\mathbf{4}$ & $\mathbf{5}$ \\
\hline Crack size $(\mathrm{mm})$ & 0.02 & 0.04 & 0.1 & 0.2 & 0.4 \\
$\begin{array}{l}\text { Data of the first stage } \\
\text { Data of the second stage }\end{array}$ & 51020408 & 25510204 & 10204082 & 5102041 & 2551020 \\
\hline
\end{tabular}

Table 3. Crack growth life data in whole process.

\begin{tabular}{lllll}
\hline Data point of number & $\mathbf{5}$ & $\mathbf{6}$ & $\mathbf{7}$ transition point & $\mathbf{8}$ \\
\hline Crack size $(\mathrm{mm})$ & 0.5 & 0.6 & 0.789 & 1.0 \\
Data of the first stage & 2040816 & 1700680 & 1293293 & 1020408 \\
Data of the second stage & 4851966 & 2859513 & 1292431 & 650026 \\
\hline
\end{tabular}

Table 4. crack growth life data in whole process.

\begin{tabular}{llllll}
\hline Data point of number & $\mathbf{9}$ & $\mathbf{1 0}$ & $\mathbf{1 1}$ & $\mathbf{1 2}$ & $\mathbf{1 3}$ \\
\hline Crack size $(\mathrm{mm})$ & 1.5 & 2.0 & 3.0 & 4 & 5 \\
Data of the first stage & 680272 & 510204 & Invalid section & \\
Data of the second stage & 200570 & 87085 & 26871 & 11667 & 6108 \\
\hline
\end{tabular}

(5) To depict the life curves in the whole process

By means of the data in tables 2-4 mentioned above have depicted the life curves for two stages and whole course are respectively in figure 2 and 3.
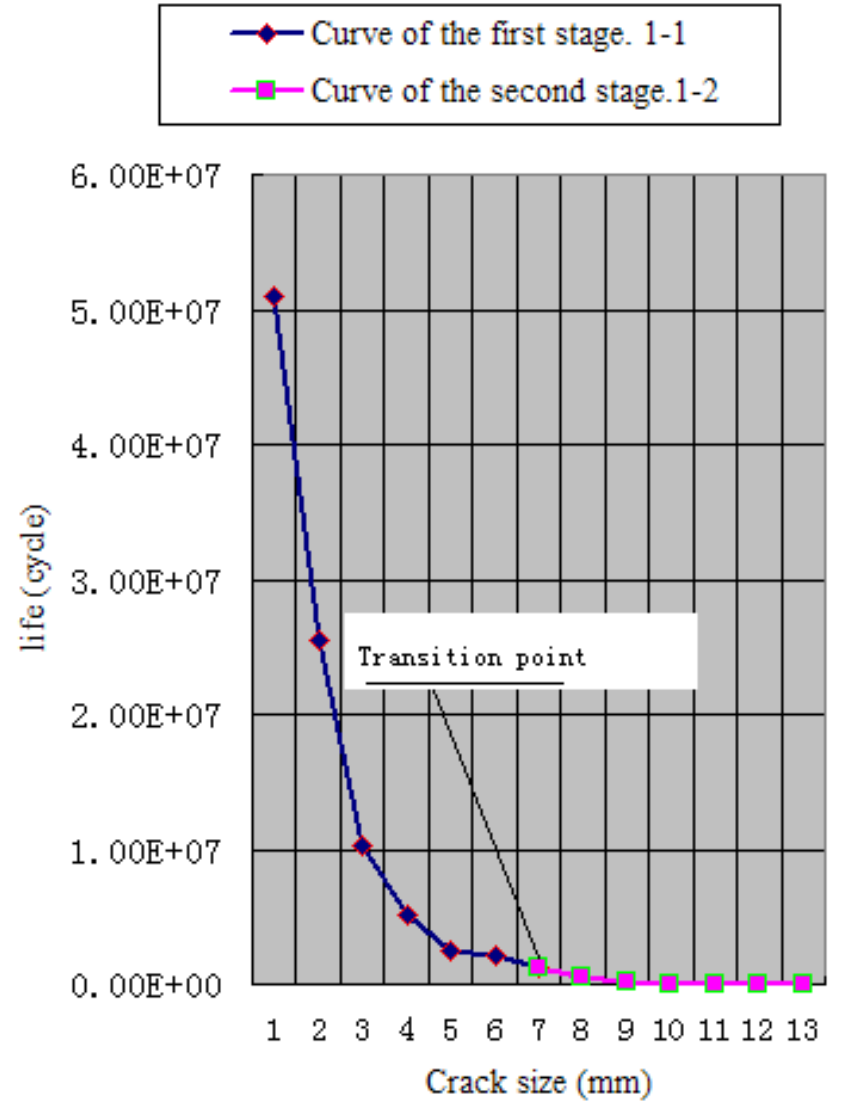

Figure 2. life curve in whole course (in decimal coordinate system).

(A) 1-1--- data curve in first stage obtained by single-parameter calculating method;

(B) 1-2---data curve in second stage obtained by single-parameter calculating method;

(C) This example transition point from micro-crack size $0.02 \mathrm{~mm}$ to long crack size 5 is just at seventh point (crack size $0.789 \mathrm{~mm}$ ).
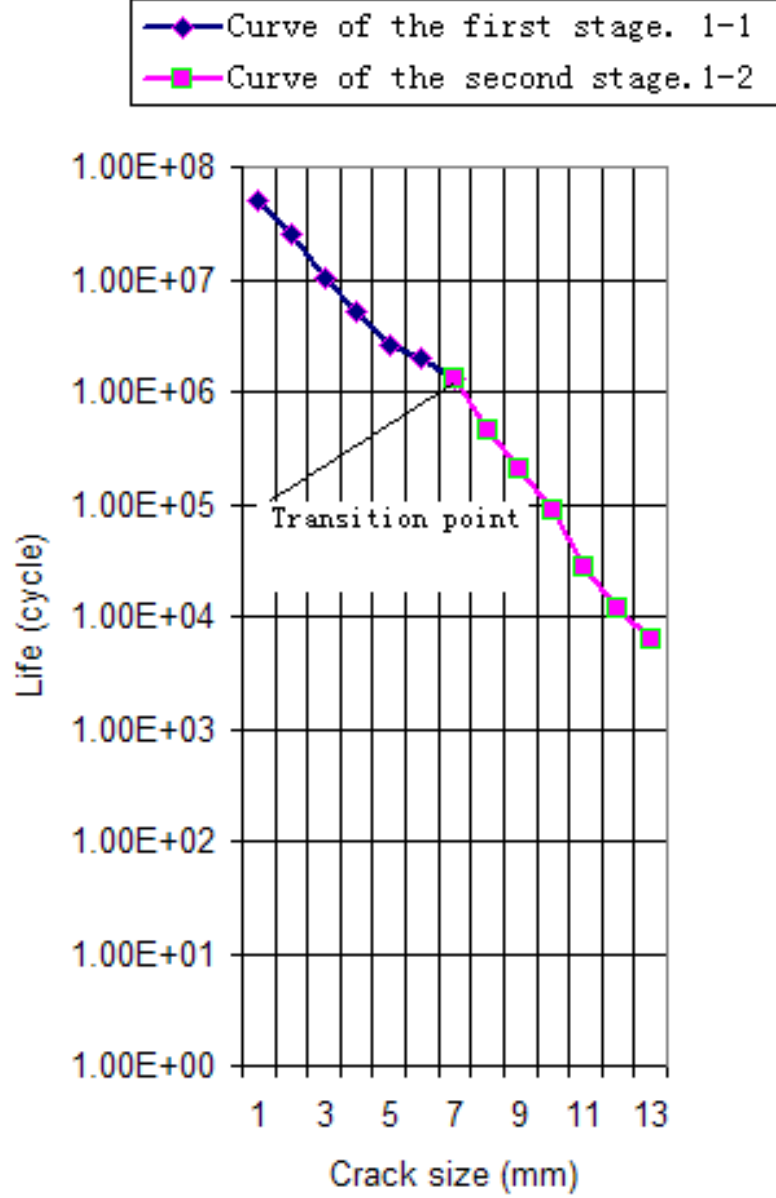

Figure 3. life curve in whole course (in logarithmic coordinate system).

(A) 1-1--- data curve in first stage obtained by single-parameter calculating method;

(B) 1-2---data curve in second stage obtained by single-parameter calculating method;

(C) This example transition point from micro-crack size $0.02 \mathrm{~mm}$ to long crack size 5 is just at seventh point (crack size $0.789 \mathrm{~mm}$ ).

\section{Discussions and Conclusions}

(1) About comparison of calculating results data: The 
lifetime data calculated by the simple stress- or strain-parameter-method as compared with the two-parameter-multiplication-method in damage mechanics [3], which is basically close, the calculating models and method is also simpler than it.

(2) About new understanding for some material constants: True material constants must show the inherent characters of materials, such as the $\sigma_{s}$ and $E, \delta, \psi$ etc in the material mechanics; for instance the $\sigma_{f}$ and $\sigma_{f}^{\prime}$; $\varepsilon_{f}$, and $\varepsilon_{f}^{\prime} ; b_{1}$ and $b_{1}^{\prime} ; c_{1}$ and $c_{1}^{\prime}$ and so on in the fatigue damage mechanics; $K_{1 c}, \delta_{c}, m_{2}, \lambda_{2}$ and so on in the fracture mechanics, above which could all be checked and obtained from general handbooks. But some key materials constants $A_{1}, A_{2}$ and $B_{2}$ in the fracture mechanics which are essentially functional formulas with other parameters the $\sigma_{f}^{\prime}$ and $\varepsilon_{f}^{\prime} ; b_{1}^{\prime} ;$ and $c_{c_{1}^{\prime}}$ etc. to have functional relations, for which can all be calculated by means of the relational expressions e.g. eqns (4-5), (13,15-16), (24-26), (40-41), (45-46), etc. that has to combine experiments and to verify. Therefore for this kind of material constants can be defined as the calculable comprehensive materials constants.

(3) About cognitions to the physical and geometrical meanings for key parameters $A_{1}$ and $A_{2}, B_{2}$ : The parameters $A_{1}$ in the first stage and $A_{2}, B_{2}$ in the second stage, they are all a concept of power, just are a maximal increment value paying energy in one cycle before to cause failure. Their geometrical meanings are a maximal micro-trapezium area approximating to beeline.

(4) About the methods for crack propagation rate and lifetime calculations in whole process: Calculation for crack transition size $a_{t r}$ it can be calculated form the crack growth-rate-linking-equation (51-53) and (57); calculation for the short crack rate it should be calculated by the short crack rate-equations $\left(d a_{1} / d N_{1}\right)$ before at transition point $a_{t r}$; for the long crack rate it should be calculated via long crack-rate-equations $\left(d a_{2} / d N_{2}\right)$ after transition point $a_{t r}$. But for the lifetime calculations in whole process can be added together by life cycle number of two stages.

(5) Based on the traditional material mechanics is a calculable subject; in consideration of the conventional materials constants there are "the hereditary characters"; In view of the relatedness and the transferability between related parameters among each disciplines; And based on above viewpoints and cognitions of the (1) (4); then for the fatigue and the fracture disciplines, if make them become calculable subjects, that will be to exist possibility.

\section{Acknowledgments}

At first, author sincerely thanks scientists David Broek, Miner, P. C. Paris, Coffin, Manson, Basquin, Y. Murakami, S. Ya. Yaliema, Morrow J D, etc, who they have be included or no included in this paper reference, for they have all made out valuable contributions for the fatigue-damage-fracture subjects. Due to they hard research, make to discover the fatigue damage and crack behavioral law for materials, to form the modern fatigue, damage-, and fracture-mechanics; due to they work like a horse, make to develop the fatigue, damage-, and fracture mechanics subjects, gain huge benefits for accident analysis, safety design and operation for which are mechanical equipments in engineering fields. Particularly should explain that author cannot have so many of discovery and provide above the calculable mathematical models and the figure 1, if have no their research results.

Author thanks sincerity the Zhejiang Guangxin New Technology Application Academy of Electromechanical and Chemical Engineering gives to support and provides research funds.

\section{References}

[1] Yu Yangui, Sun Yiming, MaYanghui and XuFeng. The Computing of intersecting relations for its Strength Problem on Damage and Fracture to Materials with short and long crack. 2011); In: International Scholarly Research Network ISRN. Mechanical Engineering Volume, Article ID 876396. http://www.hindawi.com/isrn/me/.

[2] Yangui Yu. The Calculations of Evolving Rates Realized with Two of Type Variables in Whole Process for Elastic-Plastic Materials Behaviors under Unsymmetrical Cycle. Mechanical Engineering Research. Canadian Center of Science and Education 2012; 2. (2):77-87; ISSN 1927-0607(print) E-ISSN 1927-0615 (Online).

[3] Yangui Yu. The Life Predicting Calculations in Whole Process Realized from Micro to Macro Damage with Conventional Materials Constants. American Journal of Science and Technology.Vol. 1, No. 5, 2014, pp. 310-328.

[4] Yu Yangui, Xu Feng, Studies and Application of Calculation Methods on Small Crack Growth Behaviors for Elastic-plastic Materials, Chinese Journal of Mechanical Engineering, 43, (12), 240-245. (2007), (in Chinese).

[5] YU Yangui, LIU Xiang, ZHANG Chang sheng and TAN Yanhua. Fatigue damage calculated by Ratio-Method Metallic Material with small crack under un-symmetric Cyclic Loading, Chinese Journal of Mechanical Engineering, 19, (2), 312-316, (2006).

[6] YU Yangui. Fatigue Damage Calculated by the Ratio-Method to Materials and Its Machine Parts, Chinese Journal of Aeronautics, 16, (3) 157-161, (2003).

[7] Yu Yangui and LIU Xiang. Studies and Applications of three Kinds of Calculation Methods by Describing Damage Evolving Behaviors for Elastic-Plastic Materials, Chinese Journal of Aeronautics, 19, (1), 52-58,(2006).

[8] Yangui Yu, Several kinds of Calculation Methods on the Crack growth Rates for Elastic-Plastic Steels. In: 13th International Conference on fracture (ICF13), (Beijing, 2013) In CD, ID S17-045.

[9] Morrow, j. D. Fatigue Design handbook, Section 3.2, SAE Advances in Engineering, Society for Automotive Engineers, (Warrendale, PA, 1968), Vol. 4, pp. 21-29. 
[10] Y. Murakami, S. Sarada, T. Endo. H. Tani-ishi, Correlations among Growth Law of Small Crack, Low-Cycle Fatigue Law and ,Applicability of Miner's Rule, Engineering Fracture Mechanics, 18, (5) 909-924, (1983).

[11] S. V. Doronin, et al., Ed. RAN U. E. Soken, Models on the fracture and the strength on technology systems for carry structures, (Novosirsk Science, 2005) , PP. 160-165. (in Russian)

[12] S. Ya. Yaliema, Correction about Paris's equation and cyclic intensity character of crack, Strength Problem.147, (9) 20-28(1981). (in Russian)

[13] Xian-Kui Zhu, James A. Joyce, Review of fracture toughness (G, K, J, CTOD, CTOA) testing and standardization, Engineering Fracture Mechanics, 85, 1-46, (2012).
[14] U. Zerbst, S. Beretta, G. Kohler, A. Lawton, M. Vormwald, H.Th. Beier, C. Klinger,I. C erny', J. Rudlin, T. Heckel a, D. Klingbeil, Safe life and damage tolerance aspects of railway axles - A review. Engineering Fracture Mechanics. 98, 214-271 (2013).

[15] Yu Yangui, MaYanghuia, The Calculation in whole Process Rate Realized with Two of Type Variable under Symmetrical Cycle for Elastic-Plastic Materials Behavior, in: 19th European Conference on Fracture, (Kazan, Russia, 26-31 August, 2012), In CD, ID 510 .

[16] Yu. Yangui, Bi Baoxiang, MaYanghau, Xu Feng. Damage Calculations in Whole Evolving Process Actualized for the Materials Behaviors of Structure with Cracks to Use Software Technique. In: $12^{\text {th }}$ International Conference on Fracture Proceeding. Ottawa, Canada. 2009; 12-19. CD. 